\title{
Enhancing Reuse of Data and Biological Material in Medical Research: From FAIR to FAIR-Health
}

\author{
Petr Holub, ${ }^{1}$ Florian Kohlmayer, ${ }^{2}$ Fabian Prasser, ${ }^{2}$ Michaela Th. Mayrhofer, Irene Schlünder,,3 \\ Gillian M. Martin, ${ }^{4}$ Sara Casati, ${ }^{5}$ Lefteris Koumakis, ${ }^{6}$ Andrea Wutte, ${ }^{1}$ ukasz Kozera, ${ }^{7}$ Dominik Strapagiel, ${ }^{8}$ \\ Gabriele Anton, ${ }^{9}$ Gianluigi Zanetti, ${ }^{10}$ Osman Ugur Sezerman, ${ }^{11}$ Maimuna Mendy, ${ }^{12}$ Dalibor Valík, ${ }^{13}$ \\ Marialuisa Lavitrano,, Georges Dagher, ${ }^{14}$ Kurt Zatloukal, ${ }^{15}$ GertJan B. van Ommen,, ${ }^{16}$ and Jan-Eric Litton ${ }^{1}$
}

The known challenge of underutilization of data and biological material from biorepositories as potential resources for medical research has been the focus of discussion for over a decade. Recently developed guidelines for improved data availability and reusability - entitled FAIR Principles (Findability, Accessibility, Interoperability, and Reusability) — are likely to address only parts of the problem. In this article, we argue that biological material and data should be viewed as a unified resource. This approach would facilitate access to complete provenance information, which is a prerequisite for reproducibility and meaningful integration of the data. A unified view also allows for optimization of long-term storage strategies, as demonstrated in the case of biobanks. We propose an extension of the FAIR Principles to include the following additional components: (1) quality aspects related to research reproducibility and meaningful reuse of the data, (2) incentives to stimulate effective enrichment of data sets and biological material collections and its reuse on all levels, and (3) privacy-respecting approaches for working with the human material and data. These FAIR-Health principles should then be applied to both the biological material and data. We also propose the development of common guidelines for cloud architectures, due to the unprecedented growth of volume and breadth of medical data generation, as well as the associated need to process the data efficiently.

Keywords: FAIR (Findable, Accessible, Interoperable, and Reusable) principles, provenance information management, privacy protection, open science, quality, incentives

\section{Introduction}

ThEFFICIENT SHARING OF DATA ${ }^{1}$ generated from public 1 funding and increasing dependence of research domains on data have led to the development of specific guidelines such as the FAIR data principles: Findable, Accessible, Interoperable, and Reusable. ${ }^{2}$ While the FAIR principles (Fig. 1) are a good starting point, applicable to various domains of science, they are not specific enough to deal with the major challenges of medical research, namely reproducibility and privacy

\footnotetext{
${ }^{1}$ BBMRI-ERIC, Graz, Austria

${ }^{2}$ Technical University of Munich, Munich, Germany.

${ }^{3}$ TMF e.V., Berlin, Germany.

${ }^{4}$ BBMRI.mt and University of Malta, Msida, Malta.

${ }^{5}$ BBMRI.it and Universita degli Studi di Milano-Bicocca, Milano, Italy.

${ }^{6}$ BBMRI.gr and Foundation for Research and Technology-Hellas, Heraklion, Greece.

${ }^{7}$ BBMRI.pl and Wroclaw Research Centre EIT+, Wroclaw, Poland.

${ }_{9}^{8}$ BBMRI.pl and University of Łódź, Łódź, Poland.

${ }^{9}$ Helmholtz Zentrum München, Munich, Germany.

${ }^{10}$ BBMRI.it and CRS4, Pula, Italy.

${ }^{11}$ BBMRI.tr and Acibadem University, Istanbul, Turkey.

${ }^{12}$ BBMRI.IARC and International Agency for Research on Cancer, Lyon, France.

${ }^{13}$ BBMRI.cz and Masaryk Memorial Cancer Institute, Brno, Czech Republic.

${ }^{14}$ INSERM, Paris, France.

${ }^{15}$ BBMRI.at and Medical University Graz, Graz, Austria.

${ }^{16}$ BBMRI.nl and Leiden University Medical Center, Leiden, Netherlands.

(C) Petr Holub et al., 2018; Published by Mary Ann Liebert, Inc. This Open Access article is distributed under the terms of the Creative Commons License (http://creativecommons.org/licenses/by/4.0), which permits unrestricted use, distribution, and reproduction in any medium, provided the original work is properly cited.
} 
FIG. 1. FAIR data principles.

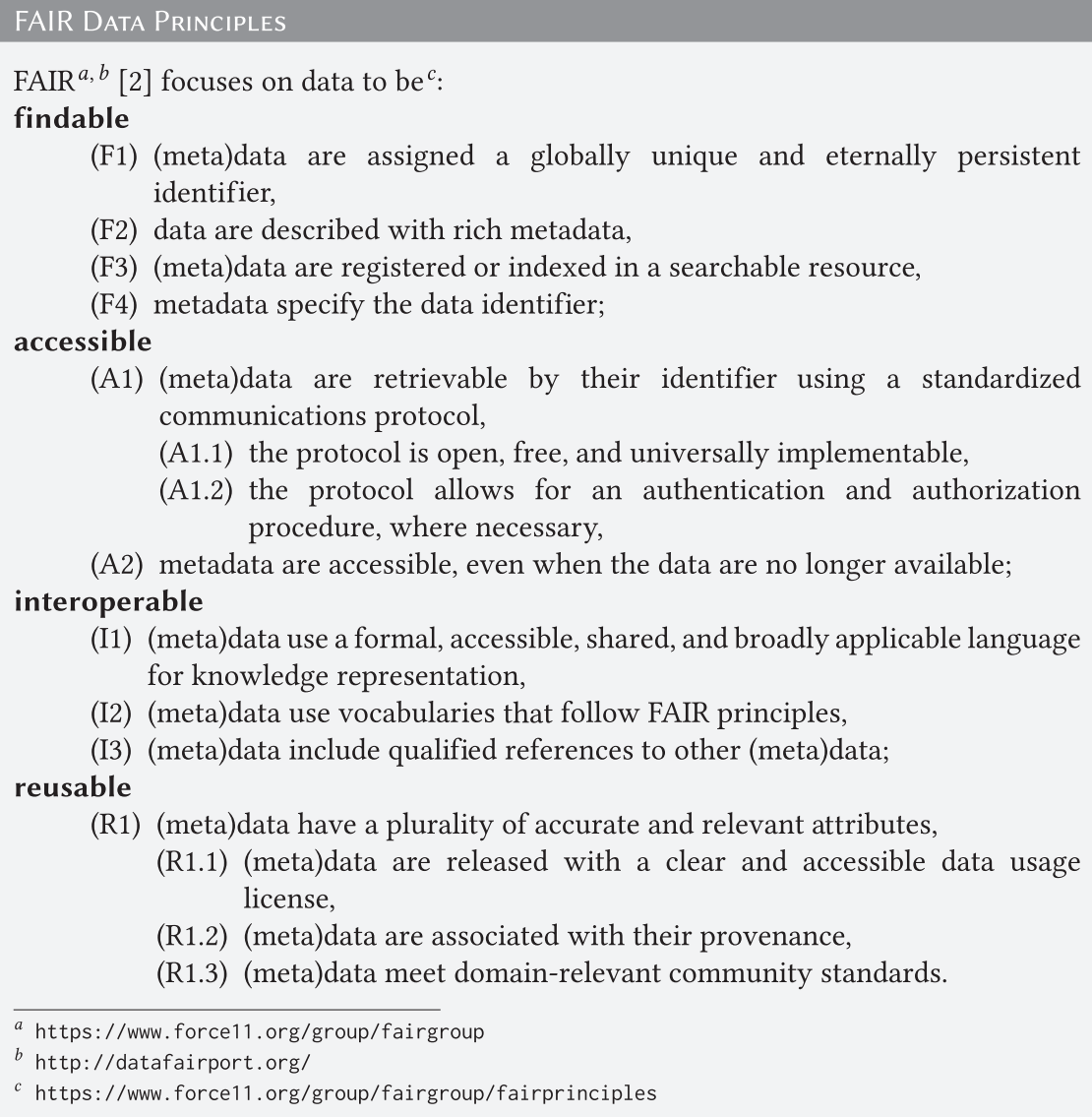

(A1) (meta)data are retrievable by their identifier using a standardized communications protocol,

(A1.1) the protocol is open, free, and universally implementable,

(A1.2) the protocol allows for an authentication and authorization procedure, where necessary,

(A2) metadata are accessible, even when the data are no longer available; interoperable

(I1) (meta)data use a formal, accessible, shared, and broadly applicable language for knowledge representation,

(I2) (meta)data use vocabularies that follow FAIR principles, reusable

(I3) (meta)data include qualified references to other (meta)data;

(R1) (meta)data have a plurality of accurate and relevant attributes,

(R1.1) (meta)data are released with a clear and accessible data usage license,

(R1.2) (meta)data are associated with their provenance,

(R1.3) (meta)data meet domain-relevant community standards.

\footnotetext{
a https://www. force11.org/group/fairgroup

${ }^{b}$ http://datafairport.org/

${ }^{c}$ https://www. force11.org/group/fairgroup/fairprinciples
}

protection. We propose a unified view of biological material and data together with specific extensions to the FAIR data principles, to boost their use and reuse in medical research.

These FAIR-Health principles include the following three main components: (1) quality and traceability; (2) incentive schemes; and (3) privacy regulation compliance. FAIRHealth principles are also applicable to other fields dealing with health-related and sensitive personal data, such as social sciences.

\section{Biobanks: A Unified View of Biological Material, Expertise, and Data}

The European Biobanking and BioMolecular resources Research Infrastructure, BBMRI-ERIC, ${ }^{*}$ anticipates substantial potential in the ability of biobanks to completely integrate the chain, from research participants and their data/ samples all the way to research results (Fig. 2).

Biobanks consist of the following key components: (1) repositories of biological material retrieved as samples from the research participants; (2) metadata describing repositories and stored samples; (3) data accompanying the samples (medical records, including imaging data and lifestyle data); (4) data generated from the samples (e.g., omics data and imaging data); (5) expertise in various fields related to longterm preservation and analyses of biological material and data, ethical/legal expertise; and (6) additional services

${ }^{*}$ www.bbmri-eric.eu related to biobanks as infrastructure (e.g., sample hosting, processing, and curation). ${ }^{3-5}$ Many biobanks are intended as facilities for long-term use, and thus research can additionally benefit from the extensive possibilities of longitudinal sample and data acquisition, such as samples/data from the same donor at different time points for studies concerning disease or treatment markers.

With current analytic and data collection techniques capable of generating almost unlimited amounts of data on each research participant donating samples and data, the question naturally arises as to whether it is meaningful, sustainable, and even technically feasible to collect, validate, store, and curate all the data on a long-term basis, and whether the volume of data is what will make medical and biomedical research more productive in the future.

As part of the strategy to address these issues, BBMRIERIC proposes a unified view of biological material and the data generated from the material, since biological material can be considered biological data storage/a biological data source. The biological material and data custodians can thus apply different strategies when combining storage to achieve acceptable costs: large raw data may be understood as transient data that can be regenerated if the original material is still available and its integrity is preserved in the long-term storage; hence, only relatively small resulting data may be kept together with the original material. We additionally argue that the high-level principles of FAIR-Health should also be applied to the biological material. The only specific aspects of biological material are that it varies in its properties and is depletable (except 


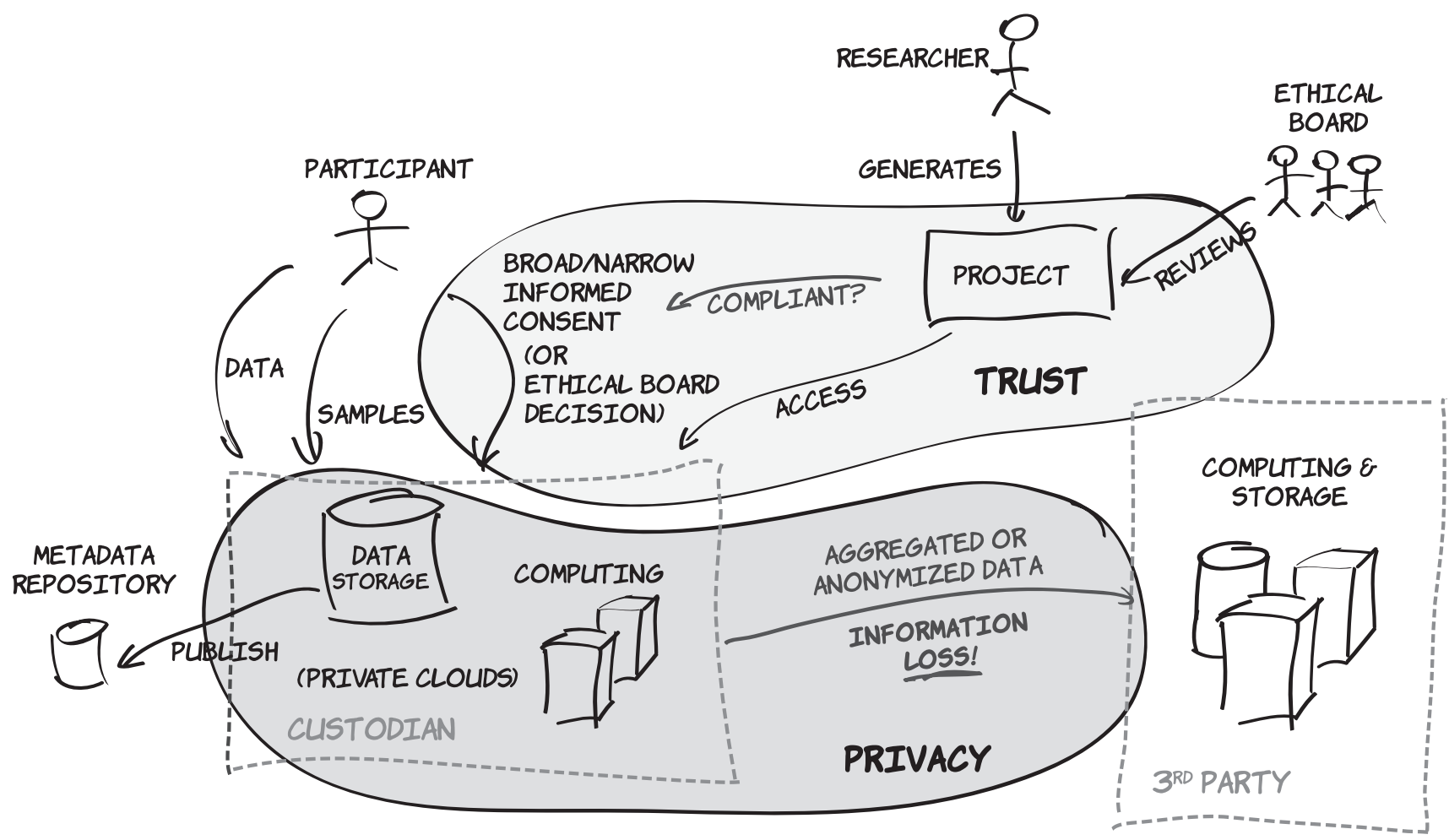

FIG. 2. The flow of data and samples from the research participants to biobanks, and then to researchers. Note the particular aspect of matching informed consent provided by the research participant to the research project, which can also be substituted by an ethical board decision if consent is not available.

for amplifiable derivatives, such as cell cultures or DNA), and therefore of limited accessibility (namely subject to access prioritization).

Translation of research results to medically meaningful knowledge and products suffers from substantial reproducibility issues. ${ }^{6-13}$ Recently, this has stimulated the proposal of countermeasures that are technically infeasible, such as an idea to store all the intermediate data used for publications ${ }^{14}$, Rule 5 . Although recognizing that primary biological material and data have to be stored for the sake of "reconstructing" results, which need to comply with principles of Good Research Practice, ${ }^{\dagger}$ it is, however, not practical and sometimes not even feasible to store all the data, due to the sheer volume of all the intermediate data. It can even be argued that storing intermediate data is of limited use when exploring reproducibility of the results, for example because of undocumented or proprietary formats.

Furthermore, reproducibility ${ }^{15}$ needs a clear link from the research participant (or other sources of nonhuman biological material), through the physical material and all the preanalytical steps, including sample stabilization and biobanking, to the data generated and published. Such links can be implemented using provenance information management systems ${ }^{16}$ (see Fig. 3 for background information on provenance). Incorporation of the preanalytical steps as a part of data provenance is increasingly recognized ${ }^{17}$ because the preanalytical phase has major implications on the

${ }^{\dagger}$ www.esf.org/fileadmin/Public_documents/Publications/Code_ Conduct_ResearchIntegrity.pdf in particular also deals with data management in Good Research Practice (section 1.4) point 1, and Guidelines for Good Practice Rules (section 2.3) point 1. preservation of biomolecules, and thus on meaningfulness of the data generated by the analysis of biological samples. For example, RNA analysis of a biological material can be subject to the checks of integrity of RNA molecules and thus the overall conclusion may be that the results are meaningful. However, the biological material, which is still "alive," may have responded to the artificial environmental conditions after its removal from its original environment, such as after a surgical resection of a tumor $\$ 18$, Annex A.

As a consequence, the material analyzed no longer represents the original material and its biological activity in the human body. While the analysis of such material is then performed in a technically correct manner, the results might not be meaningful. Accordingly, a prerequisite to obtain meaningful data requires an assessment of fitness of the biological material, based on the provenance information, for the purpose of the specific research and analytical method. The reproducibility and the meaningfulness jointly determine the quality of the biological material and data (Fig. 4). The advantage of infrastructures providing a unified view of physical material and data, such as biobanks, is that they can integrate provenance information naturally.

As part of quality improvement efforts, it is also necessary to document syntax, semantics, and history of data as completely as possible. This is particularly important for types of data that have been collected primarily for the purpose of healthcare, but can still be reused for research as a "secondary use," namely lawful use that requires fulfilling

This behavior has been documented, for example, in EU FP7 SPIDIA project (www.spidia.eu) and has become part of Annex A of CEN/TS 16826-1:2015. ${ }^{18}$ 
FIG. 3. Provenance information.
PROVENANCE INFORMATION

"Provenance is information about entities, activities, and people involved in producing a piece of data or thing, which can be used to form assessments about its quality, reliability or trustworthiness [46]." This concept is used in archival science, archeology and paleontology, computer science, bioinformatics, business processes, and other domains. Due to the need for interoperability of provenance information, there are a number of standards in these fields including a widely applicable PROV standard by $\mathrm{W}_{3} \mathrm{C}^{a}$ based on generic Entity/Agent/Activity model; Dublin Core provenance in libraries $^{b}$; Common Workflow Language ${ }^{c}(\mathrm{CWL})$ proposed within Global Alliance for Genomics and Health (GA4GH) to describe computational workflows applied to data in order to track provenance of data sets; and ISO 8000 series [47] to describe data quality of enterprise master data (key business objects) using provenance, amongst others. Interoperable provenance has also become one of elements of Electronic Health Records, as shown by HL7 FHIR Provenance ${ }^{d}$ for example.

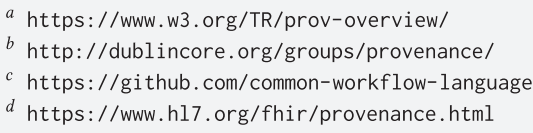

specific regulatory requirements, such as specifying anticipated secondary uses in the informed consent, or obtaining approval from an ethics committee for the reuse of data under specific conditions. While the data generation adheres to medical and laboratory best practices at any given moment, technology advances, methods evolve, and instruments are upgraded to ensure the best standard of healthcare over time.

These changes may result in vast problems when integrating the data into consistent data sets, particularly as semantics of data changes over time and across various sources, such as biorepositories, cohorts, or laboratories. These problems stimulate the need for data models and formats that can unambiguously capture syntax and semantics of the data, such as numeric data with defined notation, units, and semantics of the data, as well as methods used to generate such data. ${ }^{\S}$

\section{Provenance information management}

Complete provenance information of any biological material and data (Fig. 5) is important to interpret the data or to enrich an existing biological material and data set consistently. This provenance information must include a link to the source biological material and-if possible-a link to the information on the very research participant who donated the material.

Fragmentation of healthcare information standards, particularly in clinical settings, may pose significant impediments to this process in practice.

For certain cases, it may be necessary to develop robust distributed provenance information schemes, so that on one hand it is possible to reconstruct the whole trail, while also keeping the process compliant with data protection regulations on the other. An example of such a process could be reporting data back in case of incidental findings or if a research participant exercises his/her right to receive the data generated from them. Due to privacy protection requirements, this may involve collaboration between multiple entities responsible for different parts of the provenance information.

${ }^{\S}$ These developments are already reflected in efforts such as ISO TC 276 (Biotechnology) Working Group 5 on data integration.
Q-1: Provenance information must be FAIR. This involves development and adoption of domain-specific standards for provenance based on commonly accepted provenance information data models.

Q-2: Provenance information must continuously cover the whole chain from sample to data, ideally even from the research participant to sample, while also being compliant with data protection regulations.

\section{Quality as a prerequisite for extensibility}

To develop comparable specimen and data collections, it is important to describe the processes of obtaining and manipulating the sample from the research participant to the storage of the biological material (sometimes informally called "from the needle to the freezer"). For reproducibility reasons, it is also necessary to document all the preanalytical and analytical methods used to generate the data, as has been demonstrated in the literature. ${ }^{10,17,19}$

Q-3: Provenance information must have sufficient technical ability to describe compliance of the biological material with common quality standards, such as preanalytical standards (e.g., ISO or CEN standards).

Q-4: Provenance information must include information about analytical methods and tools used to generate data from the biological material.

\section{Incentives}

In contrast to many other scientific fields, medical data can only be made available to researchers because of voluntary contribution from a variety of individuals, particularly research participants (donors and patients) and medical doctors. A positive incentive scheme must be developed and adopted in wide research communities, which will maximize biological material and data sharing and achieve actual reuse. Effective incentive schemes will also exert pressure on resource providers to implement transparent access policies and reduce the fragmentation of access procedures. Similar incentive principles should also be applied to software tools and their sustainability, which is fundamental for any data-driven medical research. 


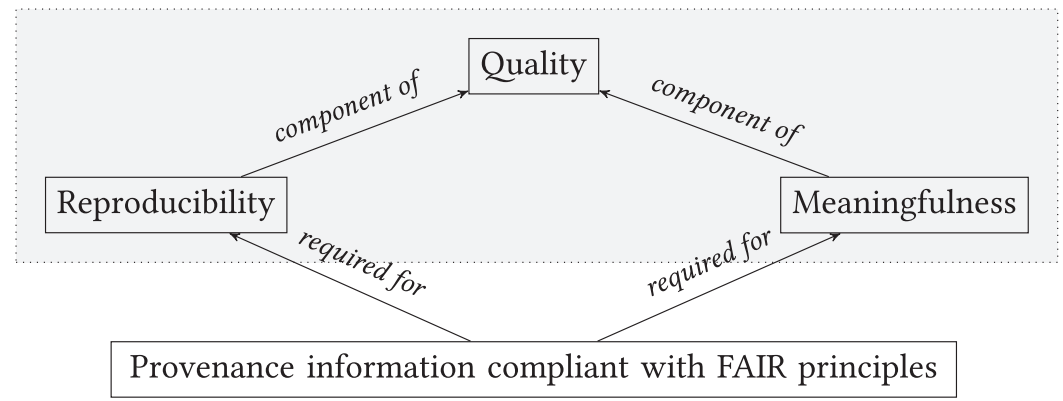

FIG. 4. Relationship between provenance information and components of quality for biological material and data.
The incentives schemes must implement the following principles:

INCE-1: Incentives must be in place for all the links in the chain: (1) biological material and data generation or collection; (2) biological material and data storage, curation, and enrichment; and (3) biological material and data reuse.

INCE-2: For biological material and data collections receiving public funding or infrastructural funding, the incentive must stimulate reuse by external users, namely users outside the infrastructure.

INCE-3: Contributions to existing biological material and data collections should be supported by funding organizations.

INCE-4: Academic promotion schemes and institutional evaluation schemes should incorporate contributing to and reuse of existing biological material and data collections.

Curation of biological material and data in INCE-1 needs to be understood as a more complex process, and not just the updating of data formats. This includes increasing its extent, as well as the enriching existing biological material and data with other types of material. Similar principles should also be applied to software tools given that medical research is dependent on the long-term availability and maintenance of high-quality software tools for data processing.

Reuse of biological material and data needs to include incentives for both sides, namely providers and accessors. Not only should researchers have incentives to use existing resources but resource providers also need clear incentives to promote and facilitate the reuse of their resources. Enforcing these principles by funding bodies, publishers, and academic organizations will exert pressure on resource providers to implement transparent access policies and make their resources more easily accessible to demonstrate their reuse. Furthermore, this should help to reduce the fragmentation of access procedures, which are effectively preventing reuse and integration of resources on a larger scale, as well as in biobanking. ${ }^{20,21}$

There is ongoing active development of metrics related to these incentive systems, as witnessed by altmetrics** by $\mathrm{NISO}^{\dagger \dagger}$ or CASRAI, ${ }^{\dagger+}$ and proposals to implement transient credit with JSON-LD. ${ }^{22}$ Micro-attribution schemes have also been suggested to support acknowledgment of contributing to large genomics data sets. ${ }^{23,24}$ These are further supported by the development of technical procedures on how to reference resources, such as BRIF $^{25}$ and the CoBRA guideline ${ }^{26}$ for biomedical and health resources. However, to date none of these systems have been widely adopted in practice.

\footnotetext{
**https://en.wikipedia.org/wiki/Altmetrics

†www.niso.org/topics/tl/altmetrics_initiative.


}

\section{P: Privacy-Respecting Access}

Particularly in the context of human data used in medical research, there are three naturally competing interests: (1) protection of privacy of individuals contributing their personal and potentially privacy-sensitive data; (2) reuse of data to maximize return on investment into research and society; and (3) complex ownership situation and economic interests. These needs have been recognized by various medical communities, as witnessed by the efforts toward clinical trial data sharing. ${ }^{27}$

As a basis for the discussion of data protection, we need to distinguish basic data types (Figure 6). We use the European General Data Protection Regulation (GDPR) ${ }^{28}$ as a basis, given that it is the most recent regulatory framework with transnational impact and because of international research collaboration, it is very likely to have an impact on a global scale. In the following text, we will also use the term privacy-enhancing technologies for a wide range of technologies that protect informational privacy by eliminating or minimizing personal data, ${ }^{29}$ e.g., coding (DT-1b) or anonymization (DT-2).

In the medical research domain, data sets are often anonymized by reducing the precision of attribute values or by removing them entirely, ${ }^{30}$ and a number of practical methodologies and tools doing just this have recently become available. ${ }^{31,32}$ For most people, the notion of anonymity implies that the remaining data are no longer privacy sensitive because no information can be traced back to or derived about the data subjects. ${ }^{33}$ However, this is not the case for many anonymization techniques. Indeed, the frequently used simple $k$ anonymity is, for example, prone to attribute disclosure. ${ }^{34}$ Even differential privacy, which is the only approach that provides rigorous mathematically grounded privacy guarantees, assumes that data cannot be totally anonymous and remain useful at the same time. ${ }^{35}$

Thus, the term (de facto) anonymized is preferred and should not be understood as static and binary, but rather as a set of various techniques for minimizing risks of privacy breaches. $^{33}$

It needs to be stressed that (de facto) anonymization is often not needed, such as in cases where informed consent is available for the given purpose, and other privacy-enhancing technologies, such as coding, may be used in conjunction with additional organizational measures. It is the responsibility of the data custodian to estimate privacy risks and adjust the technical and organizational access conditions and procedures appropriately.

In this context, there are advantages of developing large collections of biological material and data. Often, the greater the number of individuals included in a data set that is to be privacy enhanced, (1) the less information per individual has to 
FIG. 5. The ideal complete coverage of provenance information for biological material and associated data. Provenance information is abbreviated as PI in the figure.

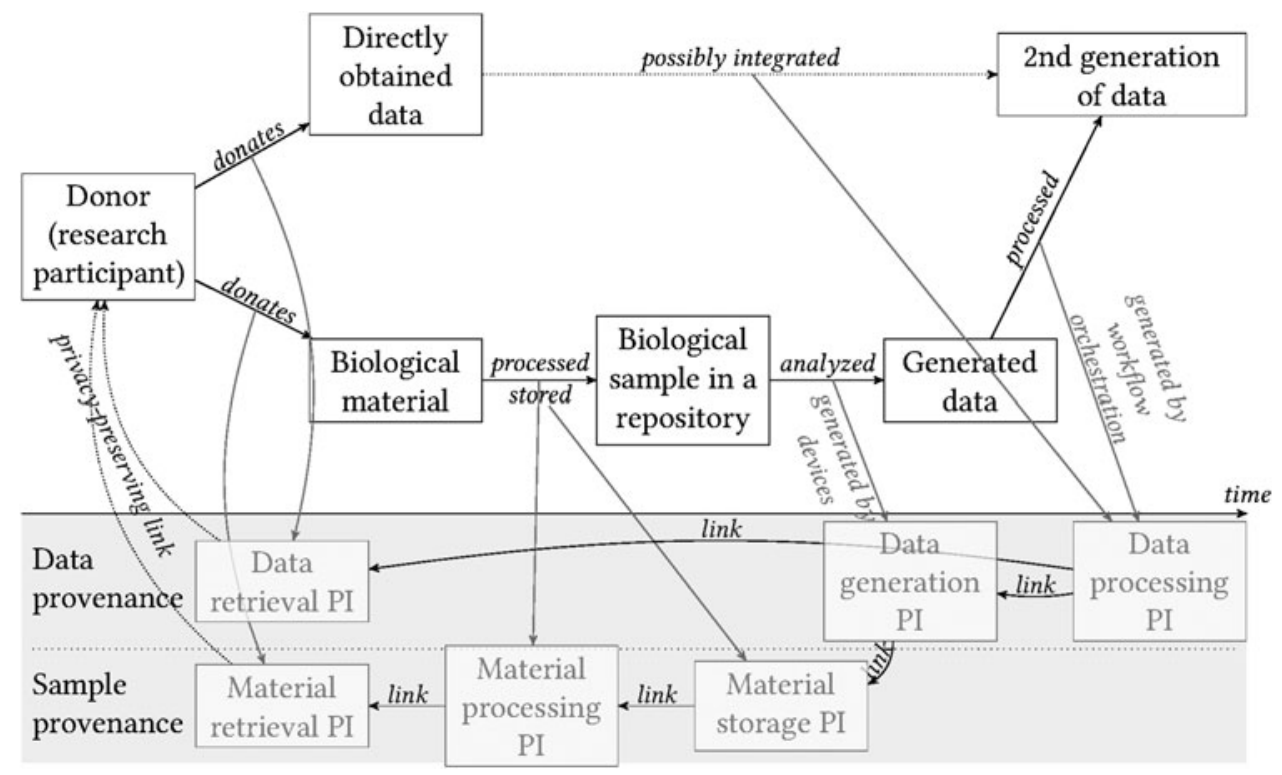

PRIVACY-RELATED DATA TYPES BASED ON GDPR

DT-1 Personal data. According to the definition of the GDPR: "personal data' means any information relating to an identified or identifiable natural person ('data subject')" [28]. This data type can be further divided into several subtypes as shown in Figure 6.

DT-1a Data directly related to individual identifiable person.

DT-1b Coded data, which typically means that some identifying information (e.g., names, civic number or social security ID) has been removed and potentially replaced with a code (a "pseudonym", but the removal of the information may not be sufficient in the sense of GDPR pseudonymization, see DT-3). This is an auxiliary type introduced in this paper, which is not directly described by the GDPR but which is often used in practice.

DT-2 (De facto) anonymized data. Anonymity of a subject from the perspective of an attacker means that the attacker cannot sufficiently identify the subject within a set of subjects, the anonymity set [48]. This data is therefore no longer personal, but it bears non-zero risk of re-identification. Anonymization must be always understood in a given context considering likelihood of attacks, e.g., from adversaries with specific background knowledge.

DT-3 Pseudonymized data. In the strict interpretation of GDPR, this is data which if the key is not known, it can be considered anonymous (i.e., with the same requirements as for DT-2).

This definition differs from previously used definitions of pseudonymization, see, e.g., [48, 49], and there is pending debate on implications of such definition (c.f. DT-1b "Coded data").

DT-4 Data from deceased people does not fall under General Data Protection Regulation but enjoys legal protection under different national jurisdictions. Furthermore professional confidentiality does not end with the death of a person (patient).

DT-5 Non-human data, which does not contain any trace of personal data and thus is privacy non-sensitive (e.g., temperature monitoring data from sample storage systems).

GDPR Article 9 defines particularly sensitive data types. Dealing with such data requires obtaining informed consent, and consequently the processing of such data must comply with the consent given. Furthermore, the research should not use any personal data, including data that receives no particular sensitivity status under GDPR, unless the data is necessary to achieve the given research purpose. 
be removed or perturbed to achieve the same residual risk of reidentification ${ }^{30,36}$ and (2) the lower the risk of reidentification when the same privacy-enhancing technology is applied.

While authors of the FAIR principles have already assumed that accessible does not always imply "open access" we introduce additional requirements to FAIR-Health to avoid overadvertising and support compliance with legal requirements:

PR-1: There must be clear identification of the responsible data controller for any given biological material and data set, who can be contacted by data subjects (research participants) or authorities.

PR-2: Compliance of (intended) research projects with informed consent and the ethics approval of the research project must be evaluated before providing access to sensitive biological material and data.

PR-3: Privacy-enhancing technologies should be applied to personal data (DT-1a) before the data can be used for research purposes, in compliance with the data minimization principle.

PR-4: Before releasing (de facto) anonymized data (DT-2), residual privacy risks, including risks of reidentification, must be considered by the data controller. The residual risks must also take into account additional safeguards, such as restricted access with sufficient level of identity assurance. ${ }^{37,38}$

PR-5: Privacy-enhancing technologies should preserve maximum value of data, while keeping the risks at an acceptable level.

The application of privacy-enhancing technologies on data to be released as a whole, as opposed to per partes on the source data subsets, should be considered. In many cases, this will minimize effects of transforming data and reduce data perturbations.

PR-6: Data provenance must be implemented in a way that allows for identification of relevant data sets in case of informed consent withdrawal. It should be noted that in case of (de facto) anonymous data (DT-2), removal of data from a specific individual may no longer be possible.

PR-7: Specifically in the case of health-related or medical data, informed consent as well as data/material transfer agreements (DTA/MTA) must define policy as to how to address incidental findings and whether access to individual's own data and results is provided, as well as how they will be accessed. In particular, the required technical and organizational safeguards have to be described.

Policies implementing PR-7 should also consider and adequately communicate that application of some privacyenhancing technologies may prevent communicating incidental findings or providing access to individual's own data/ results. This is especially important when a research project uses (de facto) anonymized data (DT-2).

Yet, no matter how high the data protection standards are set, there will always remain some risk of (re-)identification of individuals and disclosure of sensitive information about them. What is needed is the tightening of rules to protect against privacy violation that can lead to risks such as discrimination based on genetic information; the U.S. Genetic Information Nondiscrimination Act (GINA) is a good starting point ${ }^{39}$ that can be further elaborated upon. ${ }^{\S \S}$

\footnotetext{
${ }^{\S}$ GINA provides some protection against discrimination in health insurance, but it does not provide protection against discrimination in life insurance and long-term care insurance.
}

PR-8: Legal protection must be developed and implemented for individuals whose privacy has been breached accidentally or unlawfully.

\section{Privacy-respecting scalable data processing and storage}

The recent decade has seen the rise of cloud computing, ${ }^{40,41}$ allowing for various business models. As convenient as the cloud infrastructures are to achieve scalable processing and storage, they bring additional risks when used for processing data. $^{42,43}$ Several modes of operation can be implemented with respect to data protection, where data storage and processing can take place:

1. in private clouds ${ }^{41}$ built and operated by the data controller (e.g., a biobank in our case) — this enables processing of any type of data;

2. in an infrastructure that is contracted by way of a third party under such conditions that enable the third-party infrastructure to logically become part of the private infrastructure, thereby operating under the same liability for both data controller and for infrastructure provider"logically private clouds"; and

3 . in public clouds where the cloud provider does not provide any specific data protection guarantees-this is mostly restricted to data that do not require legal protection, namely (de facto) anonymized data (DT-2) with very low risk of reidentification (see requirement PR-4) or nonhuman data (DT-5).

If the data controller agrees to transfer the data to the researcher under a Material/Data Transfer Agreement (MTA/ DTA), the researcher has the same modes available. Modes 2 and 3 are nowadays subject to major ongoing development from the data protection perspective. International standards in this field recently emerged, such as ISO 27018. ${ }^{44}$ However, their adequateness and acceptance in medical research are largely open issues, hence the following additional requirements:

PR-9: Commonly-accepted policies and procurement guidelines must be developed under conditions where the thirdparty infrastructures can logically become private infrastructures suitable for storing and processing privacy-sensitive data.

PR-10: Commonly accepted guidelines must be developed for storing and processing data covered by data protection regulations on public infrastructures.

\section{Conclusions}

Life sciences generally suffer from fragmentation, while medical research in particular suffers also from substantial reproducibility issues. In this article, we proposed to extend the recently developed FAIR principles to FAIR-Health principles related to quality — namely reproducibility and meaningfulness - by providing comprehensive provenance information for the complete chain from a donor to biological material to data, as well as incentives for enriching existing resources and reusing them. Given the use of human material and data in medical research, we also propose privacyprotecting principles related to compliance with data protection regulations. European researchers, in collaboration with experts from multiple domains, including legal experts, computer science experts, and medical researchers, as well as 
research participants and citizens, are now gathering to define specific rules for a Code of Conduct for GDPR ${ }^{45}$ to ensure compliance with the regulatory frameworks. For medical research, all of these components called FAIR-Health are fundamental prerequisites for effective reuse of biological material and data.

\section{About BBMRI-ERIC}

In 2006, BBMRI was one of six Life science proposals that became part of the European Strategy Forum on Research Infrastructures (ESFRI) Roadmap for Research Infrastructures in Europe. During 2008-2011, BBMRI was funded as an EU project during the preparatory phase. After a transition phase, the Members and Associated States of the European Union approved the infrastructure as a legal entity in 2013, as a European Research Infrastructure Consortium (ERIC). Today BBMRI-ERIC is one of the largest European Research Infrastructure in the field of medicine and health. www.bbmri-eric.eu/

\section{Acknowledgments}

This work has been cofunded by ADOPT BBMRI-ERIC supported by EU Horizon 2020, Grant Agreement No. 676550; BBMRI-LPC project from the EU FP7 under Grant No. 313010; also partially supported by the grants related to the national nodes of BBMRI-ERIC, such as LM2015089 (BBMRI.cz), DIR/WK/2017/01 (BBMRI.pl), and GZ 10.470/ 0016-II/3/2013 (BBMRI.at). Authors would like to thank Jasjote Grewal for proofreading the article.

\section{Selection of Authors and Their Contributions}

The intent of the publication was announced by P.H. and J.E.L. to the Management Committee of BBMRI-ERIC and Common Services on Information Technology (CS IT), and on Ethical, Legal, and Societal Issues (CS ELSI). All the members of these bodies were invited to contribute and all the active contributors became co-authors. P.H. and J.-E.L. led the activities and preparation of the article. I.S., K.Z., and GJ.B.v.O. contributed to the overall design of the article. F.K., F.P., and I.S. contributed to the sections on privacy and data protection and I.S., G.M., M.T.M., and S.C. contributed concepts related to ethics. K.Z., D.V., and G.D. contributed to the aspects of unified view of the samples and data and to the quality section. G.Z. and U.S. contributed to the bioinformatics examples and to the concepts of privacy-protecting scalable data processing.

\section{Author Disclosure Statement}

No conflicting financial interests exist.

\section{References}

1. Problems with scientific research: How science goes wrong. The Economist 2013:13. https://www.economist.com/news/ leaders/21588069-scientific-research-has-changed-worldnow-it-needs-change-itself-how-science-goes-wrong (accessed December 29, 2017).

2. Wilkinson MD, Dumontier M, Aalbersberg IJ, et al. The FAIR Guiding Principles for scientific data management and stewardship. Sci Data 2016;3:160018.

3. Moore R. Towards a theory of digital preservation. Int $\mathbf{J}$ Digit Curation 2008;3:63-75.
4. Pennock M. Digital Curation: A life-cycle approach to managing and preserving usable digital information. For publication in Library \& Archives Journal, Issue 1, 2007. www.researchgate.net/profile/Maureen_Pennock/publication/ 228770335_Digital_curation_A_life-cycle_approach_to_ managing_and_preserving_usable_digital_information/links/ 5606535d08aeb5718ff29465.pdf (accessed December 29, 2017).

5. Albert $\mathrm{P}$, Alpi K, Baxter $\mathrm{P}$, et al. Digital research data curation: Overview of issues, current activities, and opportunities for the Cornell University Library. A report of the Cornell University Library (CUL) Data Working Group (DaWG). 2008. https://ecommons.cornell.edu/handle/1813/10903 (accessed December 29, 2017).

6. Freedman LP, Cockburn IM, Simcoe TS. The economics of reproducibility in preclinical research. PLoS Biol 2015;13: e1002165.

7. Prinz F, Schlange T, Asadullah K. Believe it or not: How much can we rely on published data on potential drug targets? Nat Rev Drug Discov 2011;10:712.

8. Begley CG, Ellis LM. Drug development: Raise standards for preclinical cancer research. Nature 2012;483:531-533.

9. Begley CG. Reproducibility: Six red flags for suspect work. Nature 2013;497:433-434.

10. AC't Hoen P, Friedländer MR, Almlöf J, et al. Reproducibility of high-throughput mRNA and small RNA sequencing across laboratories. Nat Biotechnol 2013;31:1015-1022.

11. Bissell M. Reproducibility: The risks of the replication drive. Nature 2013;503:333-334.

12. Mobley A, Linder SK, Braeuer R, et al. A survey on data reproducibility in cancer research provides insights into our limited ability to translate findings from the laboratory to the clinic. PLoS One 2013;8:e63221.

13. Morrison SJ. Time to do something about reproducibility. eLife 2014;3:e03981.

14. Sandve GK, Nekrutenko A, Taylor J, et al. Ten simple rules for reproducible computational research. PLoS Comput Biol 2013;9:e1003285.

15. Fomel S, Claerbout JF. Guest editors' introduction: Reproducible research. Comput Sci Eng 2009;11:5-7.

16. Curcin V, Miles S, Danger R, et al. Implementing interoperable provenance in biomedical research. Future Gener Comput Syst 2014;34:1-16.

17. Kap M, Sieuwerts AM, Kubista M, et al. The influence of tissue procurement procedures on RNA integrity, gene expression, and morphology in porcine and human liver tissue. Biopreserv Biobank 2015;13:200-206.

18. CEN/TS 16826-1:2015. Molecular in vitro diagnostic examinations-Specifications for pre-examination processes for snap frozen tissue-Part 1: Isolated RNA. 2015.

19. Mager S, Oomen MH, Morente MM, et al. Standard operating procedure for the collection of fresh frozen tissue samples. Eur J Cancer 2007;43:828-834.

20. Colledge F, Elger B, Howard HC. A review of the barriers to sharing in biobanking. Biopreserv Biobank 2013;11: 339-346.

21. Colledge F, Persson K, Elger B, et al. Sample and data sharing barriers in biobanking: Consent, committees, and compromises. Ann Diagn Pathol 2014;18:78-81.

22. Katz DS, Smith AM. Transitive credit and JSON-LD. J Open Res Softw 2015;3:e7.

23. The Editor. Human variome microattribution reviews. Nat Genet 2008;40:1.

24. Giardine B, Borg J, Higgs DR, et al. Systematic documentation and analysis of human genetic variation in 
hemoglobinopathies using the microattribution approach. Nat Genet 2011;43:295-301.

25. Cambon-Thomsen A, Thorisson GA, Mabile L, et al. The role of a bioresource research impact factor as an incentive to share human bioresources. Nat Genet 2011;43:503504.

26. Bravo E, Calzolari A, De Castro P, et al. Developing a guideline to standardize the citation of bioresources in journal articles (CoBRA). BMC Med 2015;13:1.

27. Institute of Medicine (IOM). Sharing Clinical Trial Data: Maximizing Benefits, Minimizing Risk. Washington, DC: The National Academies Press; 2015.

28. EU Regulation. 2016/679 of the European Parliament and of the Council of 27 April 2016 on the protection of natural persons with regard to the processing of personal data and on the free movement of such data, and repealing Directive 95/46/EC (General Data Protection Regulation). Adopted by the Council on April 8, 2016. 2016.

29. PISA Consortium. Handbook of privacy and privacyenhancing technologies. In: Van Blarkom G, Borking J, Olk J (eds). College bescherming persoonsgegevens, The Netherlands: The Hague, 2003. http://andrewpatrick.ca/ pisa/handbook/Handbook_Privacy_and_PET_final.pdf

30. El Emam K, Rodgers S, Malin B. Anonymising and sharing individual patient data. BMJ 2015;350:h1139.

31. Eder J, Gottweis H, Zatloukal K. IT solutions for privacy protection in biobanking. Public Health Genomics 2012;15: 254-262.

32. Prasser F, Kohlmayer F. Putting statistical disclosure control into practice: The ARX data anonymization tool. In: Medical Data Privacy Handbook. Springer: Charm Heidelberg New York Dordrecht, London; 2015: 111-148.

33. Rubinstein I, Hartzog W. Anonymization and risk. Available at SSRN 26461852015.

34. Sun X, Sun L, Wang H. Extended k-anonymity models against sensitive attribute disclosure. Comput Commun 2011;34: 526-535.

35. Dwork C, Roth A. The algorithmic foundations of differential privacy. Theor Comput Sci 2013;9:211-407.

36. Heatherly R, Denny JC, Haines JL, et al. Size matters: How population size influences genotype-phenotype association studies in anonymized data. J Biomed Inform 2014;52:243250.

37. Burr WE, Dodson DF, Newton EM, et al. Electronic Authentication Guideline. NIST Special Publication 800-63-2. 2013. DOI: http://dx.doi.org/10.6028/NIST.SP.800-63-2. http://nvlpubs.nist.gov/nistpubs/SpecialPublications/NIST.SP .800-63-2.pdf (accessed December 29, 2017).
38. Nenadic A, Zhang N, Yao L, et al. Levels of authentication assurance: An investigation. In: Third International Symposium on Information Assurance and Security. IEEE 2007: 155-160.

39. Feldman EA. The Genetic Information Nondiscrimination Act (GINA): Public policy and medical practice in the age of personalized medicine. J Gen Intern Med 2012;27:743-746.

40. Armbrust M, Fox A, Griffith R, et al. A view of cloud computing. Commun ACM 2010;53:50-58.

41. Mell P, Grance T. NIST SP 800-145, The NIST definition of cloud computing. 2011. DOI: 10.6028/NIST.SP.800. http:// nvlpubs.nist.gov/nistpubs/Legacy/SP/nistspecialpublication 800-145.pdf (accessed December 29, 2017).

42. Zhou M, Zhang R, Xie W, et al. Security and privacy in cloud computing: A survey. In: Semantics Knowledge and Grid (SKG), 2010 Sixth International Conference on. 2010: 105-112. DOI: 10.1109/SKG.2010.19.

43. Ryan MD. Cloud computing privacy concerns on our doorstep. Commun ACM 2011;54:36-38.

44. ISO/IEC 27018:2014-Information technology-Security techniques - Code of practice for protection of personally identifiable information (PII) in public clouds acting as PII processors. 2014.

45. Litton JE. We must urgently clarify data-sharing rules. Nature 2017;541:437.

46. W3C Working Group. PROV-Overview-An Overview of the PROV Family of Documents. Ed. by Groth, P and Moreau, L. 2013. www.w3.org/TR/prov-overview (accessed December 29, 2017).

47. ISO/TS 8000-60:2017-Data quality-Part 60: Data quality management: Overview. 2017.

48. Pfitzmann A, Hansen M. A terminology for talking about privacy by data minimization: Anonymity, unlinkability, undetectability, unobservability, pseudonymity, and identity management. Version v0.34. 2010. https://dud.inf.tu-dresden .de/literatur/Anon_Terminology_v0.34.pdf (accessed December 29, 2017).

49. ISO/TS 25237:2008-Health informatics-Pseudonymization. 2008.

Address correspondence to:

Petr Holub, PhD

BBMRI-ERIC

Neue Stiftingtalstraße 2/B/6

Graz 8010

Austria

E-mail: petr.holub@bbmri-eric.eu 